\title{
Modulation of DNA-binding activity of Mycobacterium tuberculosis HspR by chaperones
}

\author{
Correspondence \\ Sujoy K. Das Gupta \\ sujoy@boseinst.ernet.in
}

Received 7 August 2007

Revised 16 October 2007

Accepted 1 November 2007

\author{
Twishasri Das Gupta, Boudhayan Bandyopadhyay \\ and Sujoy K. Das Gupta
}

Bose Institute, Department of Microbiology, P1/12 CIT Scheme VIIM, Kolkata 700054, India

\begin{abstract}
In Mycobacterium tuberculosis, $h s p R$ is the last gene of the dnaKJE operon. It encodes the repressor HspR, which regulates the expression from this operon by binding to a consensus upstream sequence known as HAIR (HspR-associated inverted repeats). Previous investigations in the related Gram-positive bacterium Streptomyces coelicolor have revealed that DnaK acts as a co-repressor for HspR. In this investigation, a similar situation was encountered using the corresponding mycobacterial pair. However, the novel feature unearthed in this study is that the mycobacterial GroELs, GroEL1 and GroEL2, considerably stimulate the HAIR-binding activity of the HspR-DnaK combination. That these GroELs play a role in the folding process was evident from the observation that when heat- or chemically denatured HspR was renatured, the protein gained optimal activity only if one of these GroEL class chaperones was present along with DnaK. The renaturation process was found to be dependent on ATP hydrolysis. The DnaK-dependent DNA-binding activity of HspR could also be stimulated by DnaJ, but GrpE, which is known to release DnaK-bound substrates, was found to be inhibitory. The results of this study suggest that protein folding plays a substantial role in the activation of HspR following heat shock and that DnaK may be involved in two ways - first, as a chaperone acting in concert with GroEL and/or DnaJ and second, as a co-repressor bound to HspR.
\end{abstract}

\section{INTRODUCTION}

Pathogenic bacteria have to overcome various types of stress as they colonize their hosts. Understanding how the stress response operates in such bacteria is important from the overall perspective of bacterial pathogenesis. Stressresponse pathways are highly complex and involve multiple factors. Many of these factors belong to the chaperone category of proteins. It is believed that high-level expression of chaperone proteins under stress conditions is necessary for preventing irreversible loss of protein function (Lindquist \& Craig, 1988).

A substantial amount of information regarding stress tolerance pathways is available in the case of Escherichia coli, where it has been shown that the regulation is positively controlled by heat-shock sigma factors $\sigma^{32}$ (Bukau, 1993; Georgopoulos et al., 1994; Yura et al., 1993) and $\sigma^{\mathrm{E}}$ (Raina et al., 1995; Rouviere et al., 1995). Stress genes may also be negatively controlled by repressors. One such repressor, HrcA, which binds to a DNA sequence named CIRCE (controlling inverted repeat of chaperone expression) (Babst et al., 1996; Bahl et al., 1995; Schulz \& Schumann, 1996;

Abbreviations: CIRCE, controlling inverted repeat of chaperone expression; EMSA, electrophoretic mobility shift assay; HAIR, HspR-associated inverted repeats.
Segal \& Ron, 1996; Zuber \& Schumann, 1994), is the most widespread and controls the expression of dnaK and groE operons in several Gram-positive bacteria. Another wellknown regulator is $\mathrm{HspR}$, which binds to a sequence known as HAIR (HspR-associated inverted repeats) (Grandvalet et al., 1999). In Mycobacterium tuberculosis, HspR is expressed from the dnaK-grpE-dnaJ-hspR operon. HspR has been demonstrated to act as a repressor of this operon. Subsequent evidence has indicated that the expression of not only the dnaKJE genes, but also several other genes of $M$. tuberculosis, is repressed by HspR. One of these, acr2, encodes an $\alpha$-crystallin family protein which appears to be linked to virulence/pathogenesis (Stewart et al., 2005; Wilkinson et al., 2005). HspR in itself may be a virulence factor, since its knockout resulted in loss of persistence (Stewart et al., 2003). The mechanism by which this happens is not known in detail but it has been suggested that in $h s p R$ mutants, chaperones such as DnaK, which are highly antigenic, are overexpressed and this results in a better immune response in the host. However, since HspR controls several other virulence-related genes such as acr2 (Stewart et al., 2002), the overall mechanism of HspR action could be more complex than is presently thought. Binding of mycobacterial HspR to HAIR has been demonstrated using in vitro renatured protein (Stewart et al., 2001). However, this binding was not heat sensitive. It has been suggested that 
the presence of other factors could play a role in conferring heat sensitivity. In later studies, using a similar protein from Streptomyces coelicolor (Bucca et al., 2000, 2003), it was shown that DnaK acts as a co-repressor for HspR. Both these observations indicate that the activity of HspR-like regulators is possibly controlled by multiple factors, one of which is certainly DnaK.

Given the importance of $\mathrm{HspR}$ in regulating stress responses as well as virulence-related genes in M. tuberculosis, its DNA-binding activity was investigated with particular focus on the involvement of chaperones such as DnaK and GroEL in the process. The results show that whereas DnaK does indeed act as a co-repressor, as reported earlier (Bucca et al., 2000, 2003), its activity is positively modulated by GroEL and DnaJ, and negatively by GrpE.

\section{METHODS}

Bacterial strains and plasmids. E. coli strain XL1 Blue was used for routine manipulation of plasmid DNA (Sambrook et al., 1989). Mycobacterial DnaK was purified after expression from plasmid pMRLB.6 dnak/RV0350 (acquired through 'TB Research Materials \& Vaccine Testing', NIH, NIAID, based at Colorado State University) in E. coli BL21(DE3). Strains expressing mycobacterial GroELs 1 and 2 were obtained as gifts from Dr Sekhar Mande, CDFD, Hyderabad, India.

Construction of expression plasmid. The M. tuberculosis HspR expression plasmid pTD-R30 was constructed by cloning the PCRamplified $381 \mathrm{bp}$ fragment of the $h s p R$ gene (RV0353) at the BamHIHindIII site of pQE30 (Qiagen), using mycobacterial genomic DNA as template and $5^{\prime}$-CGGGATCCATGGCGAAGAACCC-3' and $5^{\prime}$ CCCAAGCTTTCACCGGCGCGGTTT-3' as forward and reverse primers, respectively. The protein expressed from the recombinant plasmid contains a stretch of six histidine residues at the $\mathrm{N}$-terminus. Clones expressing M. tuberculosis DnaJ (RV0352) and GrpE (RV0351) were constructed in the same way by cloning the respective PCR amplicons in the expression vector pQE30.

Chemicals. Chromatography material $\left(\mathrm{Ni}^{2+}-\mathrm{NTA}\right.$ agarose $)$, was purchased from Qiagen. ATP- $\gamma S$ was purchased from Calbiochem. Other chemicals for protein expression, purification and analysis were of the highest purity grade, obtained from SRL Laboratories, India. $\gamma_{-}{ }^{32} \mathrm{P}$-labelled ATP $\left(12 \times 10^{13} \mathrm{~Bq} \mathrm{mmol}^{-1}\right)$ was purchased from BRIT (Mumbai, India). Restriction enzymes and DNA-modifying enzymes, such as polynucleotide kinase and T4 DNA ligase, were obtained from New England Biolabs.

Purification of proteins. All the proteins used were N-terminally hexahistidine tagged. HspR was isolated by $\mathrm{Ni}^{2+}$-NTA agarose chromatography under denaturing conditions in the presence of $8 \mathrm{M}$ urea via a standard protocol. Briefly, E. coli cells harbouring the HspR-expressing plasmid pTD-R30 were grown up to an $\mathrm{OD}_{600}$ of 0.6 at $37{ }^{\circ} \mathrm{C}$ in $500 \mathrm{ml}$ Luria broth containing $100 \mu \mathrm{g}$ ampicillin $\mathrm{ml}^{-1}$ as antibiotic. Expression of HspR was induced by the addition of $1 \mathrm{mM}$ IPTG. Harvested cells were sonicated in lysis buffer $\left(50 \mathrm{mM} \mathrm{KH}_{2} \mathrm{PO}_{4}\right.$, $10 \mathrm{mM}$ Tris/ $\mathrm{HCl}, \mathrm{pH} 8.0,300 \mathrm{mM} \mathrm{KCl}, 8 \mathrm{M}$ urea) and centrifuged at $10000 \mathrm{~g}$. The cleared lysate was loaded on to the affinity column. The column-bound $\mathrm{HspR}$ was washed with wash buffer $\left(50 \mathrm{mM} \mathrm{KH}_{2} \mathrm{PO}_{4}\right.$, $10 \mathrm{mM}$ Tris/ $\mathrm{HCl}, \mathrm{pH} 6.3,300 \mathrm{mM} \mathrm{KCl}, 8 \mathrm{M}$ urea). The protein was then eluted using elution buffer $\left(50 \mathrm{mM} \mathrm{KH}{ }_{2} \mathrm{PO}_{4}, 10 \mathrm{mM}\right.$ Tris/ $\mathrm{HCl}$, $\mathrm{pH} 4.5,300 \mathrm{mM} \mathrm{KCl}, 8 \mathrm{M}$ urea). In some experiments, HspR was also refolded spontaneously in-column without the aid of any folding-promoting agent. This was done by washing the column with
$8 \mathrm{M}$ urea in $50 \mathrm{mM}$ Tris/ $\mathrm{HCl}, 150 \mathrm{mM} \mathrm{KCl}, \mathrm{pH} 8.0$, followed by renaturation using a $100 \mathrm{ml}$ gradient from $6 \mathrm{M}$ urea in buffer A ( $50 \mathrm{mM}$ Tris/ $\mathrm{HCl}, 150 \mathrm{mM} \mathrm{KCl}, \mathrm{pH} 8.0$ ) to the buffer alone. The renatured protein was then eluted under native conditions using

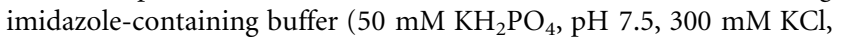
$250 \mathrm{mM}$ imidazole). The resulting protein was dialysed against phosphate buffer $\left(25 \mathrm{mM} \mathrm{KH}_{2} \mathrm{PO}_{4}, \mathrm{pH} 7.5,200 \mathrm{mM} \mathrm{KCl}\right)$ containing $15 \%(\mathrm{v} / \mathrm{v})$ glycerol. DnaK, GroEL1, GroEL2, DnaJ and GrpE were isolated through $\mathrm{Ni}^{2+}$-NTA agarose chromatography under native conditions. In the case of GroEL1, the non-ionic detergent $N$-lauryol sarcosine was added at a concentration of $10 \mathrm{mM}$ in the lysis buffer during sonication (Qamra et al., 2004).

Electrophoretic mobility shift assay (EMSA). For EMSA a $110 \mathrm{bp}$ DNA fragment derived from the DnaK promoter region $(-128$ to -38 ), encompassing two inverted HAIR repeats, was PCR amplified by using primers $\mathrm{K}-128 \mathrm{~F}$ ( $5^{\prime}$-GCTCTAGAAGCGTTAGCATGCTCAGTAAGTTG-3') and K-38R (5'-GCTCTAGATCTTTCCTGCACTATGATGAG- $\left.3^{\prime}\right)$. Ten picomoles of $\mathrm{K}-128 \mathrm{~F}$ was $5^{\prime}$ end-labelled with $\left[\gamma_{-}{ }^{32} \mathrm{P}\right]$ ATP $\left(12 \times 10^{13} \mathrm{~Bq} \mathrm{mmol}^{-1}\right)$ (BRIT) and T4 polynucleotide kinase (New England Biolabs) and directly used in PCR. The PCR product was purified using a Qiagen column. The binding reactions contained ( $30 \mu \mathrm{l}$ final volume) $3 \mu \mathrm{l} 10 \times$ binding buffer $(100 \mathrm{mM}$ Tris/HCl, pH 8, $600 \mathrm{mM} \mathrm{KCl,} 30 \mathrm{mM} \mathrm{MgCl}$, $1 \mathrm{mM}$ EDTA, $1 \mathrm{mM}$ DTT, $20 \% \mathrm{v} / \mathrm{v}$ glycerol), $1 \mu \mathrm{g}$ salmon sperm DNA, 10000 c.p.m ( $3 \mathrm{ng}$ ) labelled DNA, and the required amount of purified proteins. The reaction mixtures were pre-incubated for $5 \mathrm{~min}$ with salmon sperm DNA and then for an additional 15 min on ice, after addition of the probe. The DNA-protein complexes were separated on a $4 \%$, $40: 1$, native PAGE by electrophoresis in $0.5 \times$ Tris/borate buffer (50 mM Tris/borate, $1 \mathrm{mM}$ EDTA) at $150 \mathrm{~V}$ for $3-4 \mathrm{~h}$ at $4{ }^{\circ} \mathrm{C}$ after a pre-run at $100 \mathrm{~V}$ for $1 \mathrm{~h}$. Following electrophoresis, the gel was dried, and the bands were visualized by autoradiography. In the case of binding in the presence of chaperones, HspR was pre-incubated without salmon sperm DNA for $30 \mathrm{~min}$ in the presence of desired chaperones at specified concentrations, followed by the addition of salmon sperm DNA and then the probe.

Antibody supershift EMSA experiments were performed using specific antisera raised against the affinity-purified recombinant proteins as described earlier (Basu et al., 2002). In each case $1 \mu$ l antiserum (either immune or pre-immune) was added directly into the binding mixture followed by incubation for an additional $10 \mathrm{~min}$.

Refolding experiment. HspR $(1 \mu \mathrm{g})$ isolated under denaturing conditions was refolded in a final volume of $100 \mu \mathrm{l}$ by dilution in renaturation buffer containing $20 \mathrm{mM}$ Tris $/ \mathrm{HCl}, \mathrm{pH}$ 7.5, $1 \mathrm{mM}$ DTT, $10 \mathrm{mM} \mathrm{KCl}, 1 \mathrm{mM} \mathrm{MgCl} 2$ and $0.5 \mathrm{mM}$ ATP. In some experiments ATP was omitted or replaced by the non-hydrolysable analogue ATP$\gamma S$. Renaturation was done in either the absence or presence of the chaperones for $1 \mathrm{~h}$. After this time, aliquots $(25 \mu \mathrm{l})$ were removed and added to the DNA-binding assays. All DNA-binding assays were performed in a final volume of $30 \mu \mathrm{l}$. After $5 \mathrm{~min}$ pre-incubation in the presence of salmon sperm DNA, probe binding was done for 15 min as mentioned above. Binding was then monitored by EMSA. For a kinetic analysis, the refolding period was divided into four time intervals of $15 \mathrm{~min}$ each.

\section{RESULTS}

\section{Involvement of chaperones in the activation of HspR}

The ability of GroEL and/or DnaK to stimulate the activity of the in-column renatured protein was tested. The results 
show that, as expected, HspR did not give any binding on its own, but in the presence of the co-repressor DnaK, binding was observed (Fig. 1a). That the observed binding

a)

b)

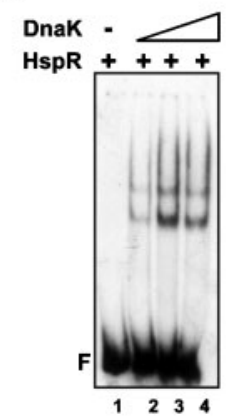

c)

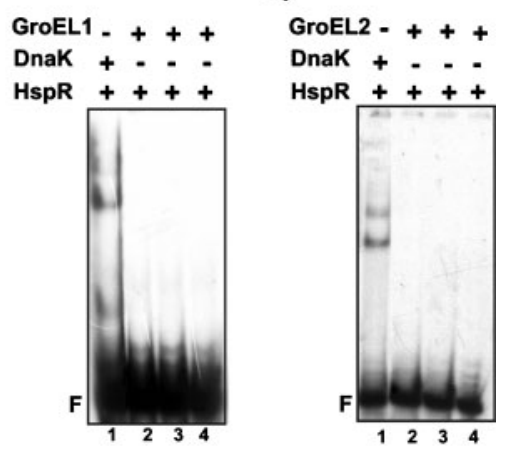

e)

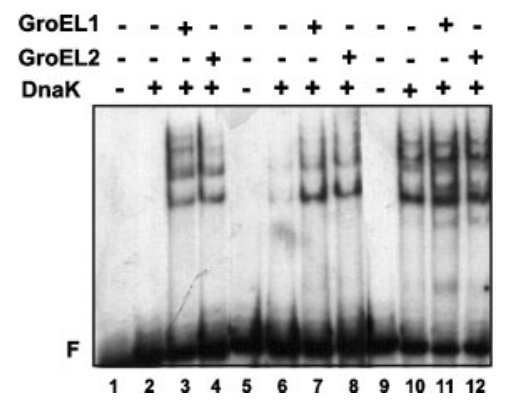

Fig. 1. Stimulation of HAIR-binding activity of in-column renatured HspR by the chaperones DnaK, GroEL1 and GroEL2 either individually or in specific combinations. (a) Binding assays were performed using $1 \mu \mathrm{g} \mathrm{HspR}$ and increasing amounts of DnaK (2.5, 5 and $7.5 \mu \mathrm{g}$ ) in a final reaction volume of $30 \mu \mathrm{l}$. (b) Binding activity in the presence of either DnaK alone ( 5 and $7.5 \mu \mathrm{g}$ ) or DnaK $(5$ and $7.5 \mu \mathrm{g})+\mathrm{HspR}(1 \mu \mathrm{g})$. (c, d) Binding activity in the presence of increasing amounts $(2.5,5,7 \mu \mathrm{g}$, lanes 2-4) of GroEL1 and GroEL2, respectively, in the absence of DnaK. Lane 1 shows binding in the presence of DnaK $(5 \mu \mathrm{g})$ but no GroEL. (e) Stimulation of the binding activity of $\mathrm{HspR}(0.25 \mu \mathrm{g}$ used in lanes $1-8$, or $1 \mu \mathrm{g}$ in lanes $9-12)$ by DnaK $(2.5 \mu \mathrm{g})$ and GroEL1 and GroEL2 (2.5 $\mu \mathrm{g}$ each). In lanes 1-4, the chaperones were added in a $1 \mathrm{~h}$ pre-incubation step in a refolding buffer, which contained $0.5 \mathrm{mM}$ ATP, whereas in lanes 5-12, the chaperones were added directly into the EMSA. In each case ' $F$ ' denotes mobility of the free probe. did not arise out of direct interaction of DnaK with the target was confirmed by performing a control experiment in which HspR was omitted (Fig. 1b, lanes 1 and 2). In contrast to DnaK, no stimulatory effect was encountered in the case of either of the mycobacterial GroELs, GroEL1 and GroEL2 (Fig. 1c and d, respectively). In order to investigate whether these GroELs play a role in the activation process in the presence of DnaK, a binding assay was set up in which the HspR concentration was lowered fourfold relative to that used in Fig. 1(a) and the DnaK concentration was kept at the lowest level. Under these conditions, neither HspR nor the DnaK-HspR combination showed any binding (Fig. 1e, lanes 2 and 6). GroEL1 and GroEL2 were then added either under refolding conditions (in the presence of ATP and pre-incubation in the presence of chaperones for $1 \mathrm{~h}$ ) or directly into the binding assay. The results (Fig. 1e, lanes 3, 4, 7 and 8) show significant stimulation by both the GroELs irrespective of the manner in which they were used. GroEL requirement, however, appeared to be essential only under limiting conditions. This was evident from the activity observed using a higher concentration of HspR (Fig. 1e, lanes 1012). In this case, no additional stimulatory effect was observed with the GroELs over and above that obtained in the presence of DnaK.

\section{Refolding denatured HspR}

The next question addressed was whether the mycobacterial GroELs have any role to play in the activation of the heat-denatured protein. HspR $(1 \mu \mathrm{g})$ was heated at $42{ }^{\circ} \mathrm{C}$ for $15 \mathrm{~min}$ and then refolded in the presence of $0.5 \mathrm{mM}$ ATP, DnaK $(10 \mu \mathrm{g})$ and increasing amounts of GroEL1. The results of this experiment (Fig. 2a) showed that the presence of GroEL1 aided the refolding of heat-denatured HspR in a dose-dependent manner. Maximum activity was encountered when the molar ratio between DnaK and GroEL1 was approximately $1: 1$. The reactivation kinetics of heat-treated HspR was then investigated either with DnaK alone or with DnaK in the presence of GroEL1 or GroEL2. The results showed that within the stipulated time of $1 \mathrm{~h}$, DnaK failed to reactivate HspR, but in the presence of either of the GroELs, a time-dependent increase in activity was observed (Fig. 2b, lanes 5-8 and 9-12). These results confirm that the presence of GroEL is essential for efficient refolding of HspR.

\section{ATP requirement for reactivation of HspR}

To test the requirement for ATP, either chemically or heatdenatured HspR (Fig. 3a and b, respectively) was allowed to refold in the presence of DnaK alone or in combination with one of the two GroELs. Activity of the refolded protein was then judged by binding assays. The results showed that in the absence of chaperones there was little or no activity (Fig. 3a and b, lanes 1). If renatured in the presence of DnaK alone, a low level of reactivation was observed, particularly in the case of the heat-denatured 
a)

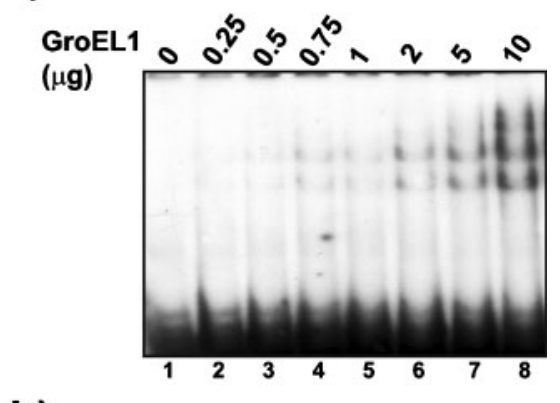

b)

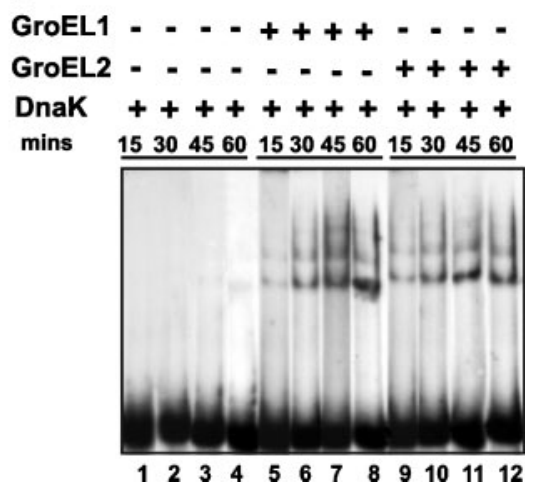

Fig. 2. (a) Dose-dependent activation of heat-denatured HspR. In-column renatured $\mathrm{HspR}(1 \mu \mathrm{g})$ was denatured at $42{ }^{\circ} \mathrm{C}$ for $15 \mathrm{~min}$, followed by incubation under refolding conditions in the presence of either DnaK $(10 \mu \mathrm{g})$ alone, or DnaK and increasing amounts of GroEL1, as indicated. The final reaction volume in the refolding process was $100 \mu \mathrm{l}$. After incubation for $1 \mathrm{~h}, 25 \mu \mathrm{l}$ aliquots were withdrawn and added to binding assays. (b) Kinetic analysis was performed in the same way as in (a) except that the incubation period under refolding conditions was increased in a stepwise manner from $15 \mathrm{~min}$ to $1 \mathrm{~h}$ as indicated. In all cases the binding assays were done for $15 \mathrm{~min}$.

protein (Fig. 3b, lane 2). This low level of DnaK-mediated reactivation was ATP dependent (Fig. 3b, lane 2 compared to lane 3 ). The level of reactivation was, however, markedly increased in the presence of either of the two GroELs (Fig. 3a and b, lanes 8-11). This reactivation appeared to be substantially, though perhaps not absolutely, dependent on ATP (Fig. 3a and b, lanes 9 and 11 compared to 8 and 10). ATP hydrolysis seems to be an important factor, since in the presence of the non-hydrolysable ATP analogue ATP- $\gamma$ S, renaturation achieved (Fig. 3c, lanes 3 and 6 ) was at the basal level, similar to that obtained in the absence of ATP (Fig. 3c, lanes 2 and 5). To test whether DnaK becomes part of the final complex, an antibody supershift EMSA was attempted. The results showed that the antiDnaK immune serum (but not pre-immune serum) produced a distinct supershift (Fig. 3d, lane 3 compared to 2). Anti-GroEL serum failed to produce similar supershifts (Fig. 3d, lanes 6 and 9 compared to 5 and 8). These results indicated that DnaK, but not GroEL, becomes part of the final complex.

\section{Role of the co-chaperones of DnaK}

The effect of mycobacterial DnaK co-chaperones DnaJ and GrpE on DnaK-dependent reactivation of HspR activity was investigated. Addition of DnaJ along with DnaK during renaturation resulted in activation of $\mathrm{HspR}$ in a manner similar to that observed with GroEL (Fig. 4a, lanes 6-9 compared to 2-5). It may be added that, in the absence of DnaK, DnaJ did not stimulate HspR activity (data not shown). Addition of GrpE to the refolding mixture did not show any stimulation. In fact, there were indications that the presence of GrpE was inhibitory. To obtain a clear idea of the extent of inhibition, a renaturation experiment was performed in which GrpE was present along with DnaK. In this assay the amount of HspR was doubled, so that the initial binding activity was strong enough to allow detection of inhibition by GrpE. The results showed that when HspR was renatured in the presence of GrpE, its DnaK-dependent binding activity decreased sharply (Fig. 4b, lanes 2-5 compared to 1 ). This indicated that GrpE inhibits the DNA-binding capacity of the DnaKHspR combination.

\section{DISCUSSION}

Stress response can play a major role in the survival of intracellular pathogens such as $M$. tuberculosis in various ways: (a) such a response can protect the bacteria within the hostile environments of the host, thereby allowing them to grow rapidly; (b) the stress proteins may themselves contribute to pathogenesis; and (c) many stress proteins, being immunogenic, contribute to the generation of host immunity. A stress response induced in a bacterium during its invasive cycle may therefore act as a double-edged sword that can prove to be both beneficial as well as harmful.

Previous investigations into mycobacterial $\mathrm{HspR}$ appear to be limited to two studies (Stewart et al., 2001, 2002) where the consequences of the deletion of $h s p R$ were examined. Some idea about how mycobacterial HspR might function may be gathered from an investigation done on a similar protein from S. coelicolor (Bucca et al., 2000, 2003). One of the points made categorically in the case of $S$. coelicolor was that DnaK is capable of acting as a co-repressor in HspRmediated binding to the operator DNA sequence known as HAIR.

In the present study, it was shown that, as in the case of the S. coelicolor HspR, in this case too, DnaK acts as a corepressor. However, the novel aspect presented here is the stimulatory role of the GroEL class chaperones of $M$. tuberculosis, GroEL1 and GroEL2, in the process. It could be demonstrated that whereas the presence of DnaK was necessary, it was not sufficient for HspR activation. The mycobacterial GroELs, GroEL1 and 2, had a major role to 


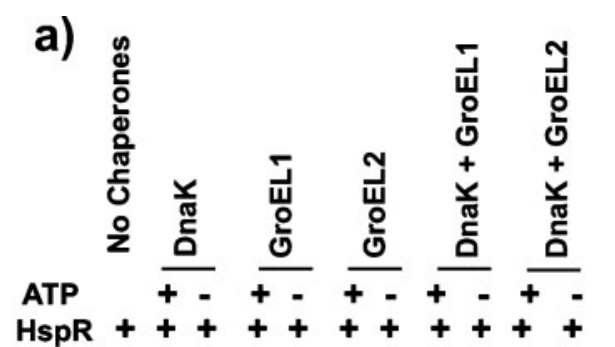

b)
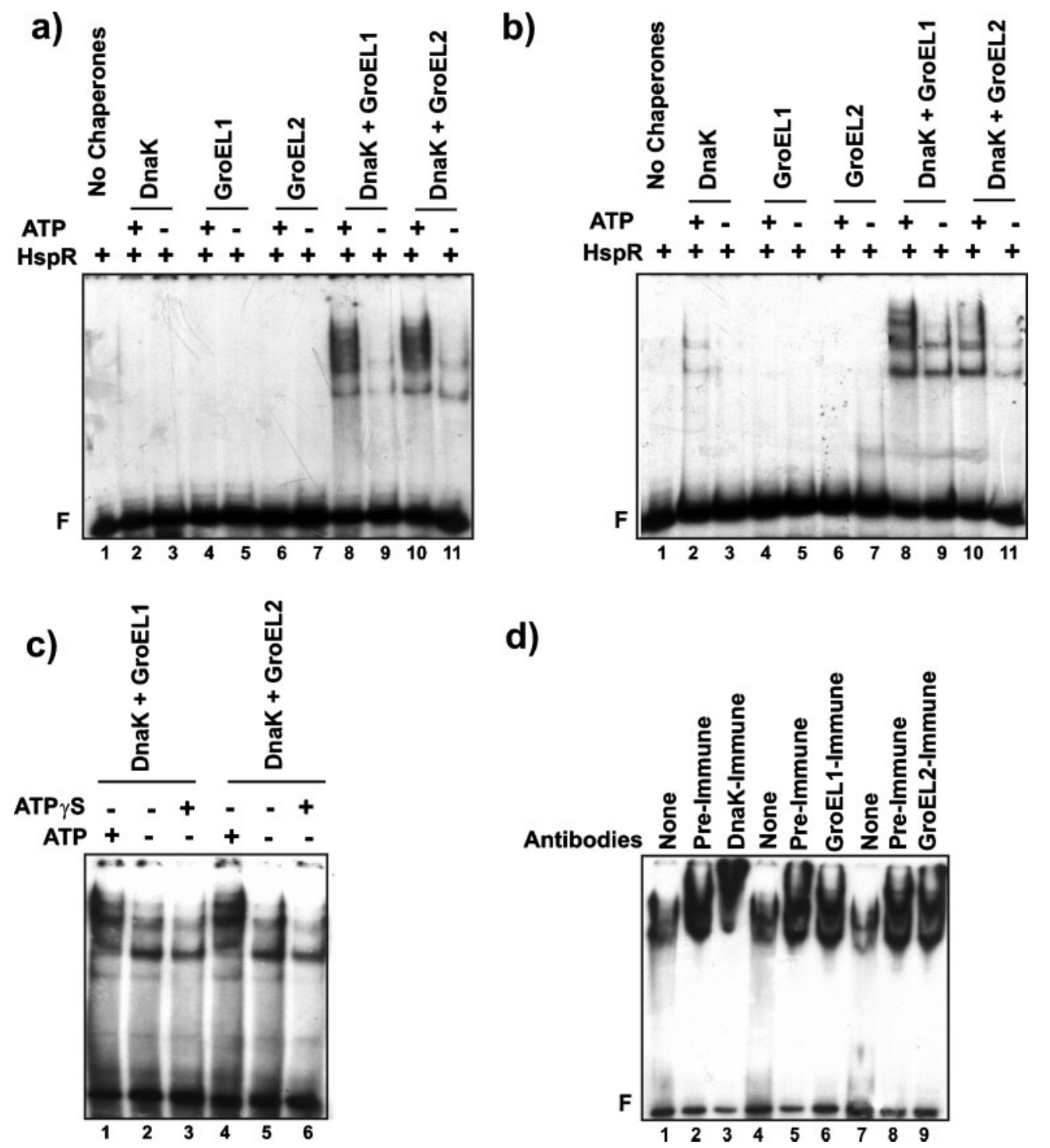

d)

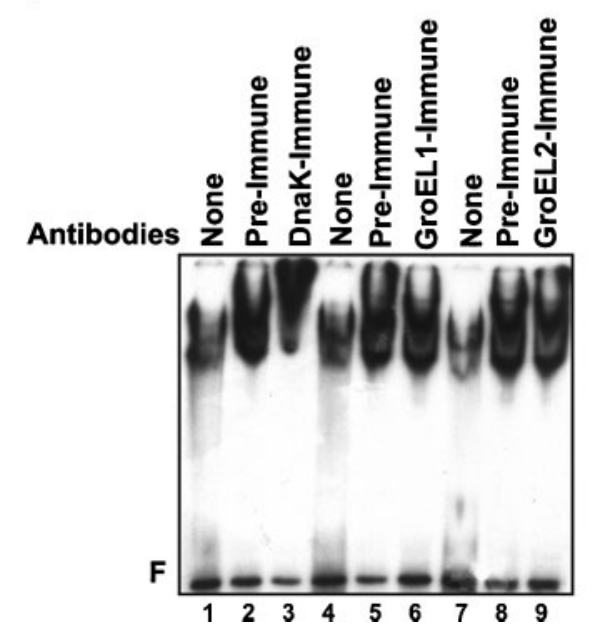

Fig. 3. (a, b) ATP requirement for the reactivation of HspR denatured either chemically (a) or by heating at $42{ }^{\circ} \mathrm{C}$ (b). Renaturation was done in either the presence or absence of DnaK $(10 \mu \mathrm{g})$ and GroEL $(10 \mu \mathrm{g})$ as indicated. ATP (0.5 mM each) was either incorporated $(+)$ or omitted (-). (c) EMSA was performed using urea-denatured HspR refolded as in (a) using either ATP or ATP $-\gamma$ S (0.5 mM each) as indicated. (d) Antibody supershift EMSA using the indicated antisera.

play in the activation processes. This co-stimulatory role of the GroELs was particularly evident when limiting amounts of HspR were used. ATP also seems to play a role, particularly in cases where the completely denatured HspR protein was refolded. Specifically, ATP hydrolysis appears to be a key event since the non-hydrolysable counterpart failed to support activation. There appears to be a degree of redundancy in the choice of co-chaperones, since DnaJ was found to be as effective as GroEL. Although the end result is the same, it is most likely that GroEL and DnaJ assist DnaK through different mechanisms. GroEL is generally known to act as a 'cage' within which a protein can fold efficiently (Mayhew et al., 1996), whereas DnaJ is known to facilitate interactions between DnaK and its substrates
(Liberek et al., 1995). The positive role played by DnaJ is on expected lines but the inhibitory effect of GrpE was unexpected. GrpE is considered to be a nucleotide exchange factor that replaces DnaK-bound ADP with ATP (Brehmer et al., 2004). ATP-bound DnaK has lower affinity for the substrate compared to the ADP-bound protein and therefore exchange of ADP with ATP by GrpE could possibly result in the release of HspR, although this has not been specifically verified. Nevertheless, this result suggests that following its successful folding, HspR must continue to be associated with DnaK in order to be active. That DnaK is indeed retained in the final DNA-protein complex was demonstrated by the antibody supershift experiment. The GroELs do not seem to be part of the 
a)

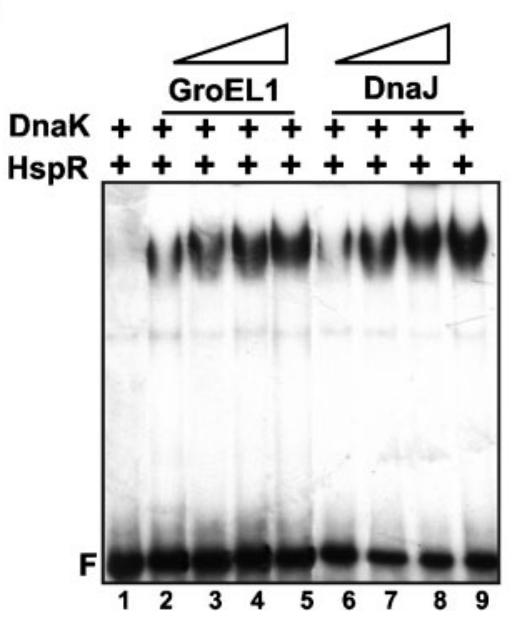

b)

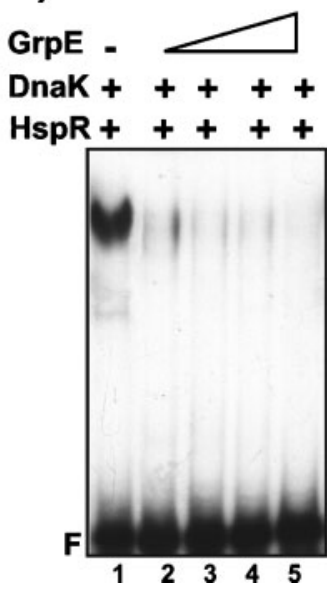

Fig. 4. Role of co-chaperones DnaJ and GrpE. (a) Binding activity of renatured HspR $(1 \mu \mathrm{g})$ in the presence of DnaK $(10 \mu \mathrm{g})$ and increasing amounts $(1,2,5$ and $10 \mu \mathrm{g})$ of either GroEL or DnaJ as indicated. (b) Same as (a) except that GrpE was used instead of DnaJ and the amount of $\mathrm{HspR}$ was $2 \mu \mathrm{g}$ instead of $1 \mu \mathrm{g}$. complex. No specific supershift was observed with the respective antiserum although their titres and specificities were comparable to that of anti-DnaK serum.

The observations presented are novel, since in the case of $S$. coelicolor HspR, activation depended solely on DnaK: neither GroEL nor ATP nor its co-chaperones had any role to play (Bucca et al., 2000, 2003). The differences in observations could be due to the simple fact that these proteins are after all derived from different systems. Alternatively, it could be due to the fact that in the previous study with the $S$. coelicor $\mathrm{HspR}$, the DnaK and GroELs used were from the unrelated bacterium E. coli, whereas in this study all the proteins used were derived from the same system, M. tuberculosis. However, the most likely reason appears to be the concentrations used. As this study shows, the stimulatory effect of the GroELs was discernible only when the HspR-binding activity was maintained at a suboptimal level.

How the mycobacterial GroELs, GroEL1 and GroEL2, assist DnaK is not clear. As mentioned earlier, GroELs are generally known to act as cages for protein folding (Mayhew et al., 1996). A complex cycle of ATP-dependent events ensures that an unfolded protein is captured and released as a completely or partly folded protein. Most of the information regarding GroEL function is derived from studies using E. coli GroEL. The role of mycobacterial GroELs in promoting protein folding in general is still not clearly understood. It has been observed that these GroELs do not require either ATP or GroES to promote protein folding (Goyal et al., 2006). Hence, in this particular scenerio the ATP dependence is unlikely to be due to the GroELs; rather it is most likely that it is DnaK that utilizes the ATP. That this is possibly the case is evident from the observation that, in some of the experiments where HspR activity was limiting, DnaK alone could activate HspR, albeit at a low level, in an ATP-dependent manner.

Since the present set of experiments strongly suggests a role for protein folding, the question may be raised as to how this phenomenon is relevant in the cellular context. To find a rationale it must be taken into account that $\mathrm{HspR}$ is a regulator of the heat-shock response. In response to heat or equivalent stress, HspR is expected to be denatured and released from the HAIR operator. However, the cell must get back quickly to its ground state. For this, HspR must attain its native structure as soon as possible, and in this context the presence of GroEL along with DnaK could be an important factor. Whether this control has anything to do with pathogenesis is a matter of conjecture, but since several virulence genes are regulated by HspR (Stewart et al., 2002), some relationship with pathogenesis is likely to emerge in the future.

\section{ACKNOWLEDGEMENTS}

We thank Dr Sekhar Mande for the two plasmids expressing the GroELs. We also thank P. Halder for technical assistance. T.D.G. is grateful to UGC, Govt of India, for her fellowship. The work was partly funded by DBT, Govt of India.

\section{REFERENCES}

Babst, M., Hennecke, H. \& Fischer, H. M. (1996). Two different mechanisms are involved in the heat-shock regulation of chaperonin gene expression in Bradyrhizobium japonicum. Mol Microbiol 19, 827-839.

Bahl, H., Muller, H., Behrens, S., Joseph, H. \& Narberhaus, F. (1995). Expression of heat shock genes in Clostridium acetobutylicum. FEMS Microbiol Rev 17, 341-348.

Basu, A., Chawla-Sarkar, M., Chakrabarti, S. \& Das Gupta, S. K. (2002). Origin binding activity of the mycobacterial plasmid pAL5000 replication protein $\mathrm{RepB}$ is stimulated through interactions with host factors and coupled expression of repA. J Bacteriol 184, 2204-2214.

Brehmer, D., Gassler, C., Rist, W., Mayer, M. P. \& Bukau, B. (2004). Influence of GrpE on DnaK-substrate interactions. J Biol Chem 279, 27957-27964.

Bucca, G., Brassington, A. M., Schonfeld, H. J. \& Smith, C. P. (2000). The HspR regulon of Streptomyces coelicolor: a role for the DnaK 
chaperone as a transcriptional co-repressor. Mol Microbiol 38, 1093-1103.

Bucca, G., Brassington, A. M., Hotchkiss, G., Mersinias, V. \& Smith, C. P. (2003). Negative feedback regulation of $d n a K, c l p B$ and lon expression by the DnaK chaperone machine in Streptomyces coelicolor, identified by transcriptome and in vivo DnaK-depletion analysis. Mol Microbiol 50, 153-166.

Bukau, B. (1993). Regulation of the Escherichia coli heat-shock response. Mol Microbiol 9, 671-680.

Georgopoulos, C., Liberek, K., Zylicz, M. \& Ang, D. (1994). Properties of the heat shock proteins of Escherichia coli and the autoregulation of the heat-shock response. In The Biology of Heat Shock Proteins and Molecular Chaperones, pp. 209-249. Edited by A. T. R. I. Morimoto \& C. Georgopoulos. Cold Spring Harbor, NY: Cold Spring Harbor Laboratory.

Goyal, K., Qamra, R. \& Mande, S. C. (2006). Multiple gene duplication and rapid evolution in the groEL gene: functional implications. J Mol Evol 63, 781-787.

Grandvalet, C., de Crecy-Lagard, V. \& Mazodier, P. (1999). The ClpB ATPase of Streptomyces albus G belongs to the HspR heat shock regulon. Mol Microbiol 31, 521-532.

Liberek, K., Wall, D. \& Georgopoulos, C. (1995). The DnaJ chaperone catalytically activates the DnaK chaperone to preferentially bind the sigma 32 heat shock transcriptional regulator. Proc Natl Acad Sci U S A 92, 6224-6228.

Lindquist, S. \& Craig, E. A. (1988). The heat-shock proteins. Annu Rev Genet 22, 631-677.

Mayhew, M., da Silva, A. C., Martin, J., Erdjument-Bromage, H., Tempst, P. \& Hartl, F. U. (1996). Protein folding in the central cavity of the GroEL-GroES chaperonin complex. Nature 379, 420-426.

Qamra, R., Srinivas, V. \& Mande, S. C. (2004). Mycobacterium tuberculosis GroEL homologues unusually exist as lower oligomers and retain the ability to suppress aggregation of substrate proteins. $J$ Mol Biol 342, 605-617.

Raina, S., Missiakas, D. \& Georgopoulos, C. (1995). The rpoE gene encoding the sigma E (sigma 24) heat shock sigma factor of Escherichia coli. EMBO J 14, 1043-1055.
Rouviere, P. E., De Las Penas, A., Mecsas, J., Lu, C. Z., Rudd, K. E. \& Gross, C. A. (1995). rpoE, the gene encoding the second heat-shock sigma factor, sigma E, in Escherichia coli. EMBO J 14, 1032-1042.

Sambrook, J., Fritsch, E. F. \& Maniatis, T. (1989). Molecular Cloning: a Laboratory Manual, 2nd edn. Cold Spring Harbor, NY: Cold Spring Harbor Laboratory.

Schulz, A. \& Schumann, W. (1996). hrcA, the first gene of the Bacillus subtilis dnaK operon encodes a negative regulator of class I heat shock genes. J Bacteriol 178, 1088-1093.

Segal, G. \& Ron, E. Z. (1996). Heat shock activation of the groESL operon of Agrobacterium tumefaciens and the regulatory roles of the inverted repeat. J Bacteriol 178, 3634-3640.

Stewart, G. R., Snewin, V. A., Walzl, G., Hussell, T., Tormay, P., O'Gaora, P., Goyal, M., Betts, J., Brown, I. N. \& Young, D. B. (2001). Overexpression of heat-shock proteins reduces survival of Mycobacterium tuberculosis in the chronic phase of infection. Nat Med 7, 732-737.

Stewart, G. R., Wernisch, L., Stabler, R., Mangan, J. A., Hinds, J., Laing, K. G., Young, D. B. \& Butcher, P. D. (2002). Dissection of the heat-shock response in Mycobacterium tuberculosis using mutants and microarrays. Microbiology 148, 3129-3138.

Stewart, G. R., Robertson, B. D. \& Young, D. B. (2003). Tuberculosis: a problem with persistence. Nat Rev Microbiol 1, 97-105.

Stewart, G. R., Newton, S. M., Wilkinson, K. A., Humphreys, I. R., Murphy, H. N., Robertson, B. D., Wilkinson, R. J. \& Young, D. B. (2005). The stress-responsive chaperone alpha-crystallin 2 is required for pathogenesis of Mycobacterium tuberculosis. Mol Microbiol 55, $1127-1137$.

Wilkinson, K. A., Stewart, G. R., Newton, S. M., Vordermeier, H. M., Wain, J. R., Murphy, H. N., Horner, K., Young, D. B. \& Wilkinson, R. J. (2005). Infection biology of a novel alpha-crystallin of Mycobacterium tuberculosis: Acr2. J Immunol 174, 4237-4243.

Yura, T., Nagai, H. \& Mori, H. (1993). Regulation of the heat-shock response in bacteria. Annu Rev Microbiol 47, 321-350.

Zuber, U. \& Schumann, W. (1994). CIRCE, a novel heat shock element involved in regulation of heat shock operon dnaK of Bacillus subtilis. J Bacteriol 176, 1359-1363.

Edited by: T. Parish 